\title{
Low thromboembolic risk for patients with the Heartmate II left ventricular assist device
}

\author{
Ranjit John, MD, ${ }^{\text {a }}$ Forum Kamdar, BS, ${ }^{a}$ Kenneth Liao, MD, ${ }^{a}$ Monica Colvin-Adams, MD, ${ }^{\mathrm{b}}$ Leslie Miller, MD, \\ Lyle Joyce, MD, and Andrew Boyle, MD $^{\mathrm{b}}$
}

\begin{abstract}
Objective: Thromboembolic events can occur in up to $20 \%$ of patients with a left ventricular assist device. The aggressive use of anticoagulation with newer continuous-flow devices has potentially increased the risk of postoperative bleeding. The predecessor of the HeartMate II left ventricular assist device, the HeartMate XVE (Thoratec Corp, Pleasanton, Calif), was associated with an extremely low thromboembolic risk, even without anticoagulation, because of its unique textured surfaces. Even though several areas of the HeartMate II are textured, a protocol was adopted for this new axial flow pump requiring long-term anticoagulation with warfarin. In our study, we investigated whether the HeartMate II left ventricular assist device is associated with a similarly low thromboembolic risk as the HeartMate XVE.
\end{abstract}

Methods: At our institution, 45 patients (mean age, $57.24 \pm 14.2$ years) underwent implantation of the HeartMate II; 30 underwent bridge-to-transplantation therapy, 7 underwent destination therapy, and 8 underwent left ventricular assist device exchange for a failed XVE left ventricular assist device. Total duration of HeartMate II support was 352.13 patient-months (mean duration, $7.2 \pm 5.2$ months). All 45 patients were treated postoperatively with warfarin and aspirin. We recorded use of these 2 medications and monthly international normalized ratios. Prospectively, we also monitored patients for any clinical thromboembolic events and for pump thrombus.

Results: Of our 45 study patients, 41 had a mean international normalized ratio of less than 2.0; of those 41 patients, 21 had a mean international normalized ratio of less than 1.6. Because of recurrent gastrointestinal bleeding episodes, 7 patients discontinued warfarin for a total duration of 39.1 patient-months. During the entire period of HeartMate II support, we noted 1 thromboembolic event. In addition, another patient had a suspected left ventricular assist device pump thrombus that resolved with a high-intensity heparin anticoagulation protocol (international normalized ratio, 1.3).

Conclusions: Our preliminary single-center analysis suggests that the HeartMate II is associated with an extremely low thromboembolic risk and with less stringent requirements for anticoagulation. Selected patients at high risk for bleeding can be safely followed with either no or extremely low anticoagulation requirements for prolonged periods.

Left ventricular assist device (LVAD) therapy is an established treatment modality for patients with refractory heart failure. ${ }^{1}$ Most patients who have undergone LVAD implantation as bridge-to-transplantation therapy in the United States have been supported with pulsatile volume displacement devices, such as the HeartMate XVE (Thoratec Corp, Pleasanton, Calif), the Novacor LVAD (WorldHeart, Oakland, Calif), or the Thoratec VAD (Thoratec Corp). ${ }^{2-6}$ Success with those devices for bridge-to-transplantation therapy led to their successful use as an alternative to transplantation (ie, as destination therapy). ${ }^{7}$ Those devices pro-

From the Division of Cardiothoracic Surgery ${ }^{\mathrm{a}}$ and the Department of Surgery and Division of Cardiology, ${ }^{\mathrm{b}}$ University of Minnesota, Minneapolis, Minn.

This study was part of the multicenter study supported by Thoratec Corp, Pleasanton, Calif.

Received for publication July 31, 2007; revisions received Nov 14, 2007; accepted for publication Dec 18, 2007.

Address for reprints: Ranjit John, MD, Division of Cardiothoracic Surgery, University of Minnesota, Minneapolis, MN 55455 (E-mail: johnx008@umn.edu).

J Thorac Cardiovasc Surg 2008; 136:1318-23

$0022-5223 / \$ 34.00$

Copyright (c) 2008 by The American Association for Thoracic Surgery doi:10.1016/j.jtcvs.2007.12.077 vide excellent hemodynamic support and improve patient survival rates, yet they do have significant constraints, including the need for extensive surgical dissection, a large body size requirement, a large-diameter percutaneous lead, and audible pump operation. Even more important, their long-term mechanical durability is limited, frequently requiring reoperations for device exchange.

The new HeartMate II LVAD, which incorporates continuous-flow rotary pump technology, represents the next generation of devices. Its focus is enhanced durability and quality of life for extended periods of circulatory support. A principal advantage of these new pumps is their small size, thereby extending therapy to underserved patient populations, including women and even some children; in addition, the HeartMate II is associated with minimal noise and greater patient comfort. ${ }^{8,9}$ The simpler design of continuousflow, rotary pump technology promises greater long-term mechanical reliability with a single moving part, the internal rotor (Figures 1 and 2). The HeartMate II LVAD is one seventh the size and one fourth the weight of the previous HeartMate XVE. We report our initial experience with changes in anticoagulation and in the risk of thromboembolism with the 


\section{Abbreviations and Acronyms}

INR = international normalized ratio

LVAD $=$ left ventricular assist device

PTT = partial thromboplastin time

TIA $=$ transient ischemic attack

HeartMate II in 45 patients with end-stage heart failure at the University of Minnesota Medical Center.

\section{MATERIALS AND METHODS \\ HeartMate II}

The HeartMate II consists of an internal blood pump with a percutaneous lead that connects the pump to an external system driver and power source (Figures 1 and 2). The pump has an implant volume of $63 \mathrm{~mL}$ and generates up to $10 \mathrm{~L} / \mathrm{min}$ of flow at a mean pressure of $100 \mathrm{~mm} \mathrm{Hg}$. Details of Heartmate II function and the surgical implant technique have been described in detail elsewhere. $^{8}$

\section{Patients}

From October 1, 2005, through May 31, 2007, a total of 45 patients (mean age, $57.24 \pm 14.2$ years) underwent HeartMate II implantation at the University of Minnesota Medical Center (30 underwent bridge-to-transplantation therapy, 7 underwent destination therapy, and 8 underwent LVAD exchange for a failed XVE model). Total duration of LVAD support was 352.1 patient-months (mean duration, $7.8 \pm 5.38$ months). All 45 patients were treated postoperatively with warfarin and aspirin. We recorded use of these 2 medications and monthly international normalized ratios (INRs). We calculated the mean INR from the mean of all the INRs recorded at monthly intervals for all patients receiving HeartMate II devices. Prospectively, we also monitored patients for any of 3 categories of clinical thromboembolic events and for device thrombosis, as defined below.

Neurologic event. Such events included any new, temporary, or permanent focal or global neurologic deficits, such as transient ischemic attack (TIA), metabolic encephalopathy, and seizure.

Stroke. A stroke is a neurologic deficit lasting more than 24 hours or lasting 24 hours or less if a brain-imaging study shows new infarction. A TIA is a neurologic deficit lasting 24 hours or less if an imaging study shows no evidence of new infarction.

Peripheral thromboembolic event. These events do not involve the central nervous system. They include a thrombus or thromboembolism in the pulmonary or systemic circulation confirmed by means of standard clinical and laboratory testing, operative findings, or autopsy findings or that requires empiric intervention.

Device thrombosis. This event entails any obstructive thrombus in the device or its conduits that is associated with clinical symptoms of impaired pump performance (eg, level 0 , none; level $1,<10 \%$ obstruction [minimal]; level $2,11 \%$ to $50 \%$ obstruction [moderate]; level 3, $>50 \%$ obstruction [severe]; and level 4, 100\% obstruction [extreme].

Anticoagulation. For our study, we agreed on this 6-step initial anticoagulation regimen as a guideline: (1) initiation of an intravenous infusion of unfractionated heparin 12 to 24 hours after HeartMate II implantation or at the point that thoracostomy tube drainage is less than $50 \mathrm{~mL} / \mathrm{h}$; (2) titration of the heparin infusion to a partial thromboplastin time (PTT) of 45 to 50 seconds for 24 hours after implantation; (3) titration of the heparin infusion to a PTT goal of 50 to 60 seconds after 24 hours; (4) titration of the heparin infusion to a PTT goal of 55 to 65 seconds after an additional 24 hours; (5) initiation of antiplatelet therapy on postoperative day 2 to 3 with $81 \mathrm{mg}$ of aspirin daily; and (6) initiation of anticoagulation with warfarin on post-

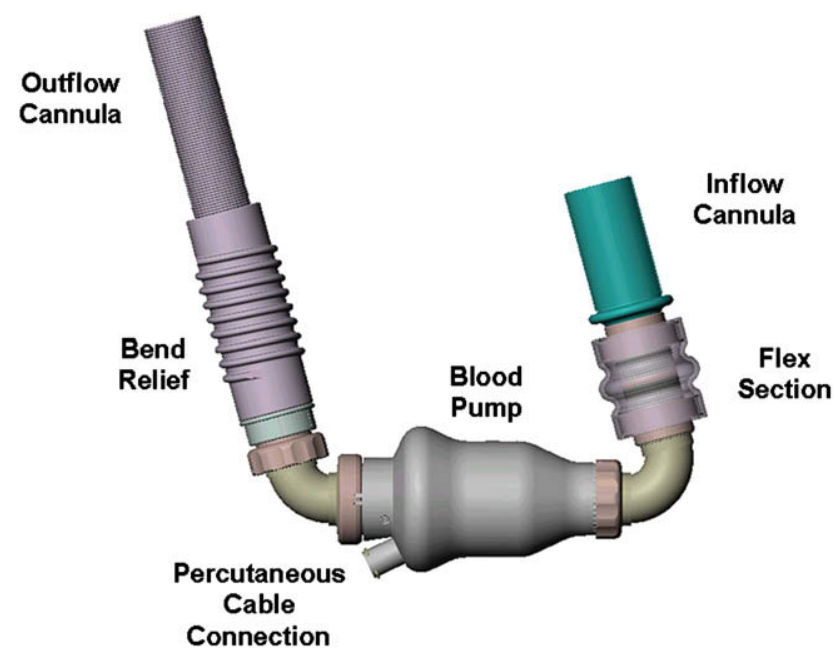

FIGURE 1. External view of the HeartMate II left ventricular assist device. Reprinted with permission from Thoratec Corp.

operative day 3 to 5 and after removal of thoracostomy tubes, titrating the dose to an INR of 2 to 3 and discontinuing heparin after obtaining a therapeutic INR.

However, as a result of significant gastrointestinal bleeding in 4 patients and delayed pericardial tamponade in 1 patient (among the first 14 patients), we decided to stop using postoperative heparin in all subsequent patients. Furthermore, we decided to initiate anticoagulation with warfarin (starting from day 3 ), titrating the dose to an INR of 1.5 to 2.0 .

\section{Data Collection}

After obtaining written informed patient consent and enrollment into the study, we collected baseline and follow-up data, including patient characteristics, results of blood chemistry analyses, hematologic findings, neurologic status, and concomitant medications. After patients were discharged from the hospital to home, they returned to our institution for follow-up, device review, and general status assessment weekly for the first 4 weeks and then monthly until the study end point. We recorded readmissions to the hospital and patient adverse events (including suspected device malfunctions) throughout the study as they occurred using standardized definitions.

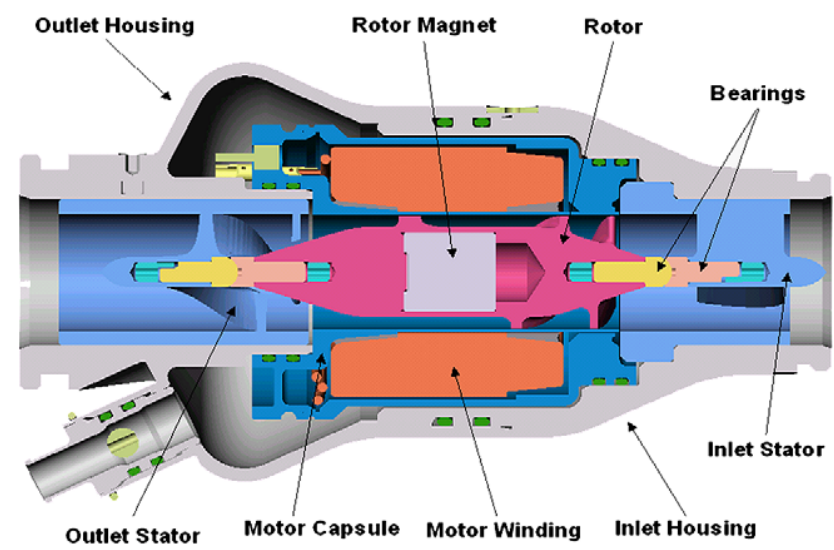

FIGURE 2. Cross-sectional internal view of the HeartMate II left ventricular assist device. Reprinted with permission from Thoratec Corp. 


\section{Statistical Analysis}

All INRs recorded for each patient for each month were averaged to obtain 1 mean value for each patient. Values are reported as means \pm standard deviations.

\section{RESULTS \\ Patient Characteristics}

Of our 45 study patients, 30 received the HeartMate II as bridge-to-transplantation therapy, 7 received it as destination therapy, and 8 received it in exchange for a failed HeartMate XVE model. The mean age of these patients was 57.24 \pm 14.2 years (range, 26-79 years), with 33 men and 12 women. The mean age of the patients in the bridge-to-transplantation group was $51.7 \pm 13.7$ years, that in the destination therapy group was $73.0 \pm 3.1$ years, and that in the failed XVE group was $64.4 \pm 9.7$ years. The cause of heart failure was ischemic in $64.4 \%(\mathrm{n}=29)$ and idiopathic in $31.1 \%(n=14)$ of all patients; 1 patient had doxorubicin (Adriamycin)-induced cardiomyopathy, and another had postpartum cardiomyopathy.

The overall mean duration of HeartMate II support was $7.2 \pm 5.2$ months, the duration in the bridge-to-transplantation group was $6.9 \pm 4.9$ months, the duration in the destination therapy group was $12.1 \pm 7.4$ months, and the duration in the XVE exchange group was $7.8 \pm 3.8$ months. The total duration of support for all patients was 352.13 patient-months.

\section{Anticoagulation}

Of our 45 study patients, 41 had a mean INR of less than 2.0; of those 41 patients, 21 had a mean INR of less than 1.6 (Figure 3). The mean INR for all 45 patients during followup is shown in Figure 4. Because of recurrent gastrointestinal bleeding episodes, 7 patients did not receive warfarin for a total duration of 39 patient-months. Of those 7 patients, 1 has been off warfarin for a total of 12 months; 2 patients have been off warfarin for 6 months. The mean INR of patients receiving warfarin (after excluding those patients not receiving warfarin) was $1.7 \pm 0.2$.

\section{Thromboembolic Events}

During the entire period of HeartMate II support, we noted only 1 thromboembolic event $(2.2 \%)$. At the time of that event, the patient had an INR of 2.4. He underwent immediate cerebral arteriography and intracerebral arterial lytic therapy (tissue plasminogen activator and mechanical embolectomy), which allowed full recovery. At the time of presentation of this thromboembolic event, this patient underwent echocardiographic analysis, which did not reveal any source of the thrombus. In addition, the patient's LVAD pump parameters (including the flow, power, and pulsatility index) did not show any abnormalities. We did not change his anticoagulation protocol; 6 weeks after this event, he was upgraded to

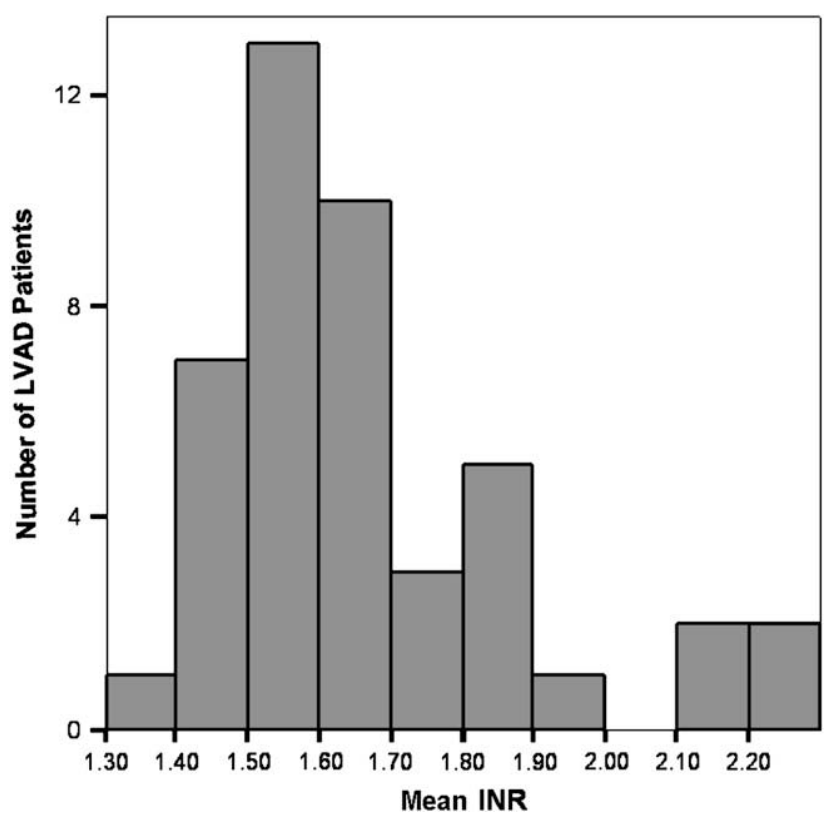

FIGURE 3. Distribution of mean international normalized ratio (INR) in HeartMate II recipients $(\mathrm{n}=45)$. LVAD, Left ventricular assist device.

United Network for Organ Sharing status IA. He subsequently underwent a heart transplantation and, as of the end of this study, is doing well. In addition, 1 patient also had postoperative paraplegia without any significant recovery to date. The cause of this event is unknown. It might have been related to this patient's perioperative hypotension. She also had immediate right ventricular failure requiring temporary right ventricular assist device support for 1 week after HeartMate II implantation with a significant pressor requirement. Thus the overall incidence of adverse neurologic events was 2 (4.4\%) of 45. Note that no TIAs occurred in this group of HeartMate II recipients. Also note that none of the patients who discontinued warfarin experienced any thromboembolic events during HeartMate II support.

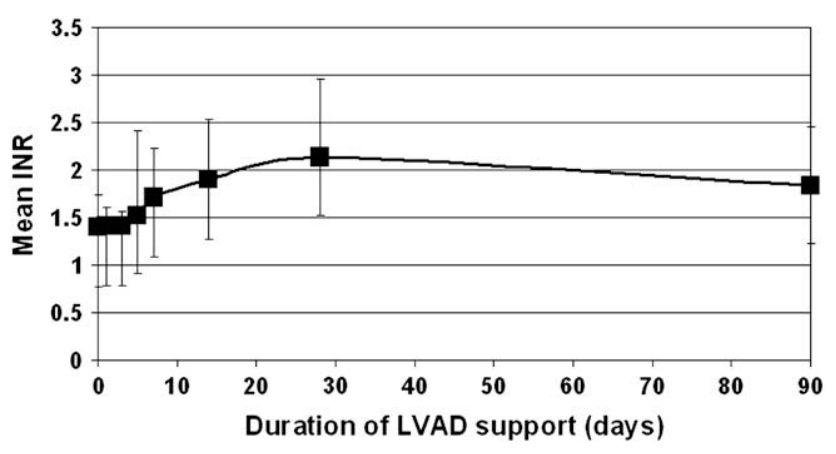

FIGURE 4. Mean international normalized ratio (INR) during HeartMate II left ventricular assist device $(L V A D)$ support $(\mathrm{n}=45)$. 


\section{Hemorrhagic Complications}

Of the first 14 patients with the HeartMate II pump in our series who received heparin followed by warfarin, 4 had gastrointestinal bleeding, and 1 had delayed pericardial tamponade $(5 / 14[35.7 \%])$. Of the remaining 31 patients who received only warfarin and no heparin, 4 had gastrointestinal bleeding, and none had pericardial tamponade (4/31 $[12.9 \%])$. Note that this analysis excluded patients who underwent mediastinal exploration for bleeding on the day of surgical intervention because that was unrelated to postoperative anticoagulation therapy.

\section{Device Thrombus}

Of our 45 study patients, 1 had a suspected pump thrombus that resolved with a high-intensity heparin anticoagulation protocol (INR, 1.3 at the time of presentation). This patient had gone to an outside hospital with HeartMate II flows in the mid-3 $\mathrm{L} / \mathrm{min}$ range (in contrast to this patient's usual flows of $>4 \mathrm{~L} / \mathrm{min}$ ) and with increased HeartMate II power. After 12 hours of high-intensity anticoagulation heparin therapy (PTT goal, 60-80 seconds), the patient's hemodynamic parameters returned to normal. Heparin therapy was continued for 48 hours. Warfarin therapy was continued to achieve an INR goal of 1.5 to 2.0. No thrombolytic therapy was used. As of the end of this study, this patient is doing well on the heart transplant waiting list without any further similar episodes 6 months after this suspected pump thrombus.

Thus, our overall incidence of device thrombus was 2 $(4.4 \%)$ of 45 , including 1 explanted pump that received a thrombus score of 3, as reported in the next section. Note that none of the patients who discontinued warfarin experienced any episodes of pump thrombus during HeartMate II support.

\section{Explanted Pump Analysis}

For the 14 patients in our study group whose HeartMate II was explanted (at the time of their heart transplantation), we analyzed their explanted pumps for thrombus deposition. All pumps except 1 received a thrombus score of 0 (none) or 1 (minimal). Figure 5 shows a typical photograph of an explanted pump with no evidence of thrombus deposition at the forward ruby bearing from a patient supported successfully by a HeartMate II for 164 days. The 1 explanted pump that received a high score of $3(>50 \%$ obstruction with thrombus) had been in a patient who died after prolonged sepsis. He had several days of low pump flow preceding his death, which might have been associated with the development of pump thrombus.

\section{DISCUSSION}

The discrepancy between the limited availability of donor organs and the ever-increasing number of patients with heart

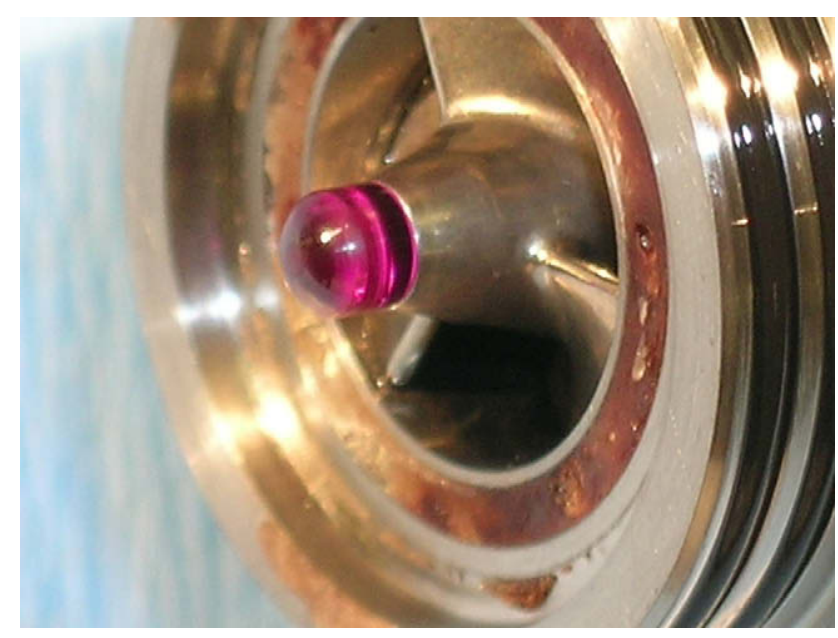

FIGURE 5. Photograph of an explanted HeartMate II LVAD with no evidence of thrombus deposition at the forward ruby bearing from a patient supported successfully for 164 days.

failure has led to the increasing use of LVADs. ${ }^{10}$ The excellent medium-term results with LVADs has led to the use of permanent LVAD implantation for patients with end-stage heart failure. ${ }^{7}$ Nevertheless, the clinical success of LVADs has been accompanied by significant complications, including thromboembolic events in as many as $30 \%$ of patients.

The development of novel materials used for implant operations and the increasing use of implanted devices has made it evident that no material is biologically inert. Commonly used biomaterials, including so-called inert compounds, such as titanium, polytetrafluoroethylene, and acrylics, can trigger an array of iatrogenic effects, including inflammation, fibrosis, coagulation, and infection. In the case of LVADs, in which the biomaterial is in direct contact with the blood circulation, significant changes in systemic immunologic and thrombostatic functions have been well documented. Like most other implanted devices, LVADs activate the coagulation system, resulting in device-related thrombus. $^{11,12}$

The standard strategy to reduce the risk of thromboembolism has been systemic anticoagulation. Despite this strategy, the risk of thromboembolism continues to be as high as $30 \%$. Furthermore, the risk of bleeding after LVAD implantation is exacerbated with anticoagulation.

The HeartMate II is a continuous-flow rotary LVAD composed of a blood pump, a percutaneous lead, and an external power source and system driver. The inlet and outlet cannulas include woven polyester grafts (CR Bard, Haverhill, $\mathrm{Pa}$ ) that require preclotting. The pump motor and associated blood tube have smooth titanium surfaces; in an effort to duplicate the excellent biocompatibility of the original pulsatile HeartMate XVE, the inlet and outlet elbows and the intraventricular cannula are textured with titanium microsphere coatings. ${ }^{8,9}$ 
The rationale for using textured materials for the original HeartMate XVE was that they would absorb and entrap elements from the patient's blood to form a stable, densely adherent biologic lining on the inside of the device. ${ }^{13-15}$ The resultant tissue lining, rather than the underlying biomaterials, would then form the long-term blood-contacting interface, thus obviating the need for systemic anticoagulation. The HeartMate XVE actually encouraged the adherence of a thin layer of fibrin-based neointima to its rough interior surfaces, yet the risk of embolization was reduced by the dense adherence of this acquired biologic lining to its surface. The physiologic consequences of this biologic lining, however, were many and varied because of the nature and characteristics of the cells adhering to the HeartMate XVE surface. Spanier and colleagues ${ }^{11,16}$ described a phenomenon of a "compensated coagulopathy" underlying the apparent autoanticoagulation in textured-surface HeartMate $\mathrm{XVE}$ recipients, attributing this finding to procoagulant stimuli elicited from the LVAD cell-surface environment. Some investigators have suggested that such activation of anticoagulation was due largely to the continuous contact of blood with the foreign LVAD surface. However, others showed that specific cells that progressively adhere to the textured LVAD surface and become activated can also contribute to the coagulopathy. ${ }^{14,16}$

In addition to the thromboembolic risks inherent with all LVADs, including the HeartMate II, the left ventricle in patients with end-stage heart failure is also prone to the development of thrombus in areas of pre-existing scar tissue, of previous myocardial infarction, and of previous ventricular aneurysm; such patients can even have a pre-existing thrombus. Thus they might already have an increased risk of thromboembolism, even before LVAD implantation. Moreover, after LVAD implantation, the degree of blood stasis in the patient's heart is determined, in part, by the opening of the native aortic valve. At our institution, we usually adjust the fixed-rate speed of the HeartMate II to maximize left ventricular decompression and to improve cardiac output, simultaneously allowing for at least a 1:3 ratio of aortic valve opening. We believe this adjustment has a favorable effect on reducing the incidence of thromboembolism associated with continuous-flow pumps, such as the HeartMate II.

In patients undergoing cardiopulmonary bypass, an association between a higher rate of microembolic signals during cardiac surgery and postsurgical neurologic dysfunction has been described. ${ }^{17,18}$ The clinical relevance of detecting microemboli is relatively unclear; this phenomenon is mostly unrelated to the development of clinically significant adverse neurologic events in patients receiving LVADs (including HeartMate II). ${ }^{19-21}$ Whether routine surveillance with transcranial Doppler monitoring confers a significant clinical benefit remains unanswered. ${ }^{22}$

Our study was limited by its relatively small number of single-center patients. Also, using the mean INR to represent the degree of anticoagulation might not truly capture the level of anticoagulation accurately. It is certainly possible that between patients' monthly clinic visits, their levels of anticoagulation might be higher or lower than what is shown when the INR is being measured.

In conclusion, the new continuous-flow HeartMate II LVAD has benefited from duplicating several features of the original HeartMate XVE, especially in that both confer a low thromboembolic risk. The explanation for the improved thromboembolic rate most probably has more to do with the influence of high pump flows, well-executed computational fluid dynamics modeling, and the continuousflow nature of the device than with the textured surfaces. The average flow in all 45 of our patients was consistently greater than $4 \mathrm{~L} / \mathrm{min}$. Such high flow is associated with reduced stasis and with better washing effect and consequently with a favorable effect on the risk of thrombus formation in both the device pump and the patient's heart. With increasing experience, we have also successfully reduced the intensity of anticoagulation (avoiding heparin and decreasing the therapeutic INR, which formerly was $2.0-3.0$ and now is 1.5-2.0), without any increased risk of thromboembolic events. In addition, we currently treat patients with lowdose warfarin therapy ( 2 to $3 \mathrm{mg} / \mathrm{d}$ ) to achieve a therapeutic INR of 1.5 to 2.0 by the end of the first week. The favorable low thrombogenicity and thromboembolic risk associated with the HeartMate II makes it well suited as destination therapy. With the increasing number of adverse reactions and the increasing intolerance to anticoagulation in the destination therapy population, our experience with avoiding anticoagulation (other than aspirin) in a select, albeit small, number of patients receiving HeartMate II LVADs further favors its use. Moreover, with the increasing number of LVAD recipients who later undergo noncardiac surgery (eg, hernia repairs and orthopedic procedures), the ability to discontinue anticoagulation without a fear of thromboembolic complications is paramount. Further studies are needed that seek to identify the molecular and biochemical markers of thromboembolism and bleeding to better understand thromboembolic and bleeding risks in LVAD recipients.

We thank Mary E. Knatterud, PhD, for editorial assistance.

\section{References}

1. Frazier OH, Rose EA, Oz MC, Dembitsky W, McCarthy P, Radovancevic B, et al. Multicenter clinical evaluation of the HeartMate vented electric left ventricular assist system in patients awaiting heart transplantation. $J$ Thorac Cardiovasc Surg. 2001;122:1186-95.

2. Deng MC, Edwards LB, Hertz MI, Rowe AW, Keck BM, Kormos RL, et al. International Society for Heart and Lung Transplantation. Mechanical circulatory support device database of the International Society for Heart and Lung Transplantation: third annual report-2005. J Heart Lung Transplant. 2005;24:1182-7.

3. Frazier OH, Rose EA, McCarthy P, Burton NA, Tector A, Levin H, et al. Improved mortality and rehabilitation of transplant candidates treated with a long-term implantable left ventricular assist system. Ann Surg. 1995;222:327-36.

4. Farrar DJ, Hill JD, Pennington DG, McBride LR, Holman WL, Kormos RL, et al. Preoperative and postoperative comparison of patients with univentricular and 
biventricular support with the Thoratec ventricular assist device as a bridge to heart transplantation. J Thorac Cardiovasc Surg. 1997;113:202-9.

5. Portner PM, Jansen PG, Oyer PE, Wheldon DR, Ramasamy N. Improved outcomes with an implantable left ventricular assist system: a multicenter study. Ann Thorac Surg. 2001;71:205-9.

6. Slaughter MS, Tsui SS, El-Banayosy A, Sun BC, Kormos RL, Mueller DK, et al. Results of multicenter clinical trial with the Thoratec implantable ventricular assist device. J Thorac Cardiovasc Surg. 2007;133:1573-80.

7. Rose EA, Gelijns AC, Moskowitz AJ, Heitjan DF, Stevenson LW, Dembitsky W, et al. Long-term mechanical left ventricular assistance for end-stage heart failure. N Engl J Med. 2001;345:1435-43.

8. Griffith BP, Kormos RL, Borovetz HS, Litwak K, Antaki JF, Poirier VL, et al. HeartMate II left ventricular assist system: from concept to first clinical use. Ann Thorac Surg. 2001;71:116-20.

9. Frazier OH, Myers TJ, Westaby S, Gregoric ID. Clinical experience with an implantable, intracardiac, continuous flow circulatory support device: physiologic implications and their relationship to patient selection. Ann Thorac Surg. 2004; 77:133-42.

10. Aaronson KD, Eppinger MJ, Dyke DB, Wright S, Pagani FD. Left ventricular assist device therapy improves utilization of donor hearts. Ann Thorac Surg. 2004; 39:1247-54.

11. Spanier TB, Oz MC, Levin HR, Weinberg A, Stomatis K, Stern D, et al. Activation of coagulation and fibrinolytic pathways in patients with left ventricular assist devices. J Thorac Cardiovasc Surg. 1996;112:1090-7.

12. Menconi MJ, Prockwinse S, Owen TA, Dasse KA, Stein GS, Lian GB. Properties of blood contacting surfaces of clinically implanted cardiac assist devices: gene expression, matrix composition, and ultrastructural characterization of cellular linings. J Cell Biochem. 1995;57:557-73.

13. Rose EA, Levin HR, Oz MC, Frazier OH, Macmanus Q, Burton NA, et al. Artificial circulatory support with textured interior surfaces: a counterintuitive approach to minimize thromboembolism. Circulation. 1994;90(suppl):II87-91.
14. Graham TR, Dasse K, Coumbe A, Salih V, Marrinan MT, Frazier OH, et al. Neointimal development on textured biomaterial surfaces during clinical use of an implantable left ventricular assist device. Eur J Cardiothoracic Surg. 1990;4: 182-90.

15. Slater JP, Rose EA, Levin HR, Frazier OH, Roberts JK, Oz MC. Low thromboembolic risk without anticoagulation using advanced design left ventricular assist devices. Ann Thorac Surg. 1996;62:1321-8.

16. Spanier TB, Chen JM, Oz MC, Stern DM, Rose EA, Schmidt AM. Time-dependent cellular population of textured-surface left ventricular assist devices contributes to the development of a biphasic systemic procoagulant response. J Thorac Cardiovasc Surg. 1999;118:404-13.

17. Clark RE, Brillman J, Davis DA, Lovell MR, Price TR, Magovern GJ. Microemboli during coronary artery bypass grafting. J Thorac Cardiovasc Surg. 1995;109: 249-58.

18. Georgiadis D, Grosset DG, Kelman A, Faichney A, Lees KR. Prevalence and characteristics of intercranial microembolic signals in patients with different types of prosthetic cardiac valves. Stroke. 1994;25:587-92.

19. Schmid C, Weyand M, Nabavi DG, Hammel D, Deng MC, Ringelstein EB, et al. Cerebral and systemic embolization during left ventricular support with the Novacor N100 device. Ann Thorac Surg. 1998;65:1703-10.

20. Thoennissen NH, Schneider M, Allroggen A, Ritter M, Dittrich R, Schmis C et al. High level of cerebral microembolization in patients supported with the DeBakey left ventricular assist device. J Thorac Cardiovasc Surg. 2005;130: 1159-66.

21. Moazami N, Roberts K, Argenziano M, Catanese K, Mohr JP, Rose EA, et al Asymptomatic microembolism in patients with long-term ventricular assist support. ASAIO J. 1997;43:177-80.

22. Wilhelm CR, Ristich J, Knepper LE, Holubkov R, Wisniewski SR, Kormos RL, et al. Measurement of hemostatic indexes in conjunction with transcranial Doppler sonography in patients with ventricular assist devices. Stroke. 1999;30:2554-61 